\title{
SATB2-AS1 acts as a miR-299-3p sponge to facilitate tumorigenesis in human non-small cell lung cancer
}

Xiaofeng Wu, Junti Lu, Wen Chen, Meng Liang, Nan Wang, Yanhui Zhou

Taihe Affiliated Hospital of Hubei University of Medicine, Taihe, China

Submitted: 30 December 2019; Accepted: 18 March 2020

Online publication: 5 April 2021

Arch Med Sci

DOI: https://doi.org/10.5114/aoms/119168

Copyright $\odot 2021$ Termedia \& Banach

\section{Abstract}

Introduction: Non-small cell lung cancer (NSCLC), the most common pathological type of lung cancer, is partly responsible for the increasing number of tumor-related deaths worldwide. This study aimed to explore the biological role of the anti-sense transcript of special AT-rich sequence binding protein 2 (SATB2-AS1), a novel cancer-related long non-coding RNA (InCRNA), and illustrate the potential molecular mechanisms.

Material and methods: The expression patterns of SATB2-AS1 were determined via qPCR analysis in clinical samples and tumor cell lines. Kaplan-Meier survival analysis was conducted to assess the relationship between SATB2-AS1 expression and survival time of NSCLC patients. NSCLC tumors were transfected with SATB2-AS1 expression vectors or specific short hairpin RNAs (sh-SATB2-AS1). Tumor cell proliferation, cell cycle progression and apoptosis were detected by MTT assays and the flow cytometric method. Nude mouse transplantation models were applied to investigate the effects of SATB2-AS1 on tumor cell growth in vivo. Bio-informatics analysis, luciferase reporter assays and rescue assays were performed to elucidate possible molecular mechanisms.

Results: SATB2-AS1 up-regulation was observed in tumorous tissues and cell lines. Up-regulated SATB2-AS1 expression was associated with shorter overall survival time of patients. SATB2-AS1 over-expression facilitated tumor cell proliferation, cell cycle progression and survival, while its knockdown inhibited tumor cell proliferation, cell cycle progression and survival. SATB2-AS1 depletion suppressed tumor growth in vivo. SATB2-AS1 was revealed to act as a miR-299-3p sponge to exert a carcinogenic effect.

Conclusions: Our data indicate that SATB2-AS1 acts as a miR-299-3p sponge to facilitate NSCLC development, providing a novel candidate therapeutic target for NSCLC.

Key words: SATB2-AS1, miR-299-3p, proliferation, apoptosis, non-small cell lung cancer.

\section{Introduction}

Recent advances in omics-based technologies have revealed that more than $90 \%$ of the mammalian genome could be transcribed, among which protein-coding genes account for only $1-2 \%$ while the vast majority of resultant transcripts are non-coding RNAs (ncRNAs) [1, 2]. It is widely accepted that ncRNAs consist of microRNAs (miRNAs) and long ncRNAs (IncRNAs) and that IncRNAs are transcribed mainly by polymerase II as single-stranded molecules [3, 4]. LncRNAs are defined as a large and diverse class of RNA transcripts with a length of over 200 nucleotides,
Corresponding author:

Yanhui Zhou

Taihe Affiliated Hospital

of Hubei University

of Medicine

Taihe, China

E-mail:jlin284@163.com 
which possess low or no protein-coding potential $[5,6]$. Numerous studies have demonstrated that IncRNAs are implicated in diverse biological processes, including cell proliferation, cell differentiation, cell survival and signaling transduction $[7,8]$. Increasing evidence has shown that IncRNAs may exert their regulatory roles via acting as guides, tethers, scaffolds or decoys of other biological molecules $[9,10]$. Recent studies have identified that the aberrant expression of some tumor-related IncRNAs contributes to non-small cell lung cancer initiation and progression [11-13].

Lung cancer is one of the most common and aggressive human malignant tumors throughout the world, resulting in over one million deaths every year $[14,15]$. It is estimated that approximately 220,000 new lung cancer cases occur and that about 160,000 people die of this cancer in the US every year $[16,17]$. Non-small cell lung cancer (NSCLC) is the most prevalent pathological type of lung cancer and accounts for around $85 \%$ of all lung cancer cases [18-21]. Chemotherapy, radiotherapy, immunotherapy and combined therapy remain the major treatment options for NSCLC patients $[22,23]$. In spite of significant progress in NSCLC diagnosis and therapy over the past decades, the prognosis of NSCLC patients is still poor, highlighting the urgent demand for novel and effective therapeutic alternatives. Notably, recent reports have pointed to the functional significance of some cancer-related IncRNAs in NSCLC development [24, 25].

The anti-sense transcript of special AT-rich sequence binding protein 2 (SATB2-AS1), a newly identified tumor-associated IncRNA, is transcribed from a region of chromosome 2 in the opposite direction to the protein-coding transcript [26]. Previous studies have characterized the biological role of SATB2-AS1 in human osteosarcoma [27] and colorectal cancer [28]; nonetheless, little is known about its role in NSCLC and underlying molecular mechanisms. In the current study, we aimed to explore the biological role of SATB2-AS1 in NSCLC and elucidate the potential molecular mechanisms involved. The present study may provide a better understanding of NSCLC development.

\section{Material and methods}

\section{Patients and tissue sample collection}

Tumorous tissue samples and adjacent normal tissue samples were collected from 60 NSCLC patients between March 2013 and February 2017 at Taihe Hospital (Taihe, China). All the participants enrolled in this study provided written informed consent, and the study obtained approval from the Medical Ethics Committee of Taihe Hospital.

\section{Cell lines, cell culture and transfection}

Four NSCLC cell lines (H661, A549, H460, Calu-1) and one normal human bronchial epithelial cell line, BEAS-2B, were purchased from the American Type Culture Collection (ATCC). All the cells were cultured in RPMI-1640 medium (Invitrogen, Carlsbad, CA, USA) and maintained at $37^{\circ} \mathrm{C}$ in a humidified atmosphere of $5 \% \mathrm{CO}_{2}$.

Short hairpin RNA specifically targeting SATB2AS1 (sh-SATB2-AS1) and miR-299-3p inhibitor were commercially constructed by GenePharma Co. Ltd (Shanghai, China). The sequence information for sh-SATB2-AS1 and miR-299-3p was listed as follows: for sh-SATB2-AS1, 5'-GCCACGAGUAUAUCAGCAACUUUCAAGAGAAGUUGCUGAUAUAACUCGUGGCUU-3'; for miR-299-3p inhibitor, 5'-UAUGUGGGAUGGUAAACCGCUU-3'. Cell transfection was performed using Lipofectamine 2000 (Invitrogen) following the manufacturer's instructions.

\section{MTT assays}

Cell proliferation was evaluated using MTT assays. In brief, cells were seeded in 96-well plates at the density of $1.5 \times 10^{4}$ per well and cultured in a humidified incubator. Following incubation for different times ( 0 h, 24 h, $48 \mathrm{~h}$ and $72 \mathrm{~h}$ ), DMSO solution (Sigma, St. Louis, MO, USA) was added to each well. Then cells were incubated for another four hours at $37^{\circ} \mathrm{C}$. The absorbance at $570 \mathrm{~nm}$ was measured using an automatic microplate spectrophotometer (Spectra Max 250; Molecular Devices, Sunnyvale, CA, USA).

\section{Cell cycle analysis}

The cell cycle was evaluated using flow cytometry analysis as previously described [29]. In brief, cells were harvested and then were washed twice using cold PBS. The cells were re-suspended in $300 \mu \mathrm{l}$ of PBS, followed by addition of $500 \mu \mathrm{l}$ of PI staining solution (50 $\mathrm{ng} / \mathrm{ml}$ ). Subsequently, the cells were incubated in the dark for 30 min at room temperature. Finally, the cells were analyzed using a FACSCalibur Flow Cytometer (BD Biosciences, Franklin Lakes, NJ, USA).

\section{Cell apoptosis analysis}

FITC-Annexin V/PI Apoptosis Detection Kit (BD Biosciences) was used for cell apoptosis analysis following the manufacturer's protocol. Briefly, cells were collected, washed twice with cold PBS, and re-suspended in $500 \mu \mathrm{l}$ of binding buffer. Following addition of $5 \mu \mathrm{l}$ of Annexin V-FITC and 5 $\mu \mathrm{PI}$, cells were incubated in the dark for $15 \mathrm{~min}$ at room temperature. Subsequently, cell apoptosis was assessed on a FACSCalibur Flow Cytometer (BD Biosciences). 


\section{Western blotting analysis}

Total cellular proteins were extracted and then were separated using SDS-PAGE as previously described [30-32], then were transferred onto PVDF membranes. The membranes were blocked using $5 \%$ non-fat milk at room temperature for $1 \mathrm{~h}$. Next the membranes were incubated with primary antibodies (Abcam, Cambridge, MA, USA) at $4^{\circ} \mathrm{C}$ overnight. Following incubation with HRP-conjugated secondary antibody, the protein bands were developed with an ECL detection kit (Amersham, Piscataway, NJ, USA).

\section{RNA isolation, reverse transcription and $\mathrm{qRT}$-PCR}

Total RNAs were extracted using the TRIzol reagent (Invitrogen) in line with the manufacturer's instructions. For miRNA analysis, reverse transcription was performed using a PrimeScript miRNA cDNA synthesis kit (Takara, Dalian, China). For IncRNA analysis, a PrimeScript Reverse Transcriptase Reagent Kit (Takara) was used to synthesize the cDNA. Quantitative PCR was carried out using the SYBR Green Master Mix on an ABI 7500 Real Time PCR System (Applied Biosystems, Foster City, CA, USA). The specific primers were designed and synthesized by GenePharma. The sequences of primers were listed as follows: for SATB2-AS1, forward 5'-CGAATCCCTTCCTCCTCTCC-3' and reverse 5'-TCGTCTTAGCCCTTTCCGTT-3'; for $\beta$-actin, forward 5'-GTGGCCGAGGACTTTGATTG-3' and reverse 5'-CCTGTAACAACGCATCTCAT-3'; for miR-299-3p, forward 5'-ACACTCCAGCTGGGTATGTG-3' and reverse 5'-GTGCAGGGTCCGAGGTCG-3'; for U6, forward 5'-CTCGCTTCGGCAGCACA-3' and reverse 5'-AACGCTTCACGAATTTGCGT-3'. U6 was used as an internal control to normalize the expression of mi299-3p. $\beta$-actin acted as an endogenous control for SATB-AS1 expression analysis. The relative expression levels were calculated using the $2^{-\Delta \Delta \mathrm{ct}}$ method.

\section{In vivo tumorigenic assays}

The animal experiment was conducted in accordance with the guidelines of the Helsinki Declaration, and was approved by the Animal Care and Use Committee of Taihe Hospital (Taihe, China). In brief, tumor xenograft models were established by subcutaneous injection of sh-NC or sh-SATB2-AS1-treated $\mathrm{H} 460$ cells in the flanks of the nude mice (5-6 weeks of age). The length and width of tumors were measured using a slide caliper every 7 days. At post-inoculation day 35 , mice were sacrificed, then tumors were harvested and weighed. The volumes of the collected tumors were calculated using the following formula: volume $\left[\mathrm{mm}^{3}\right]=$ width $^{2}\left[\mathrm{~mm}^{2}\right] \times$ length $[\mathrm{mm}] / 2$. Furthermore, tumor samples were collected for further immuno-histochemical analysis.

\section{Immunohistochemical staining}

Immunohistochemical analysis was carried out on 5- $\mu \mathrm{m}$-thick sections prepared from formalin-fixed and paraffin-embedded tissues. Tissue sections were de-paraffinized in xylene and rehydrated in a graded series of ethanol. Microwave heating was used for antigen retrieval. After being blocked using 5\% bovine serum albumin, tissue sections were incubated with rabbit Ki67 polyclonal antibody at $4^{\circ} \mathrm{C}$ overnight. Subsequently, tissue sections were treated with biotinylated secondary antibody for $1 \mathrm{~h}$ at room temperature. Diaminobenzidine (DAB) was used as a chromo gen. The Ki67-positive cells in the tumor tissues were observed under a light microscope (BX51; Olympus, Tokyo, Japan).

\section{Prediction and selection of target miRNA}

The starBase v3.0 online tool was used to predict potential target miRNAs of SATB2-AS1. Afterwards, three cancer-related miRNAs (miR-299-3p, miR-545-3p, and miR-605-3p) were selected as candidate targets of SATB2-AS1. Then, qRT-PCR analysis was conducted to evaluate whether SATB2-AS1 over-expression and knockdown could affect the expression of the above candidate miRNAs. Consequently, only miR-299-3p expression could be significantly regulated by SATB2AS1, suggesting that miR-299-3p was a potential binding partner for SATB2-AS1. Thus, miR-299-3p was chosen for subsequent research.

\section{Dual luciferase reporter assays}

The wild-type SATB2-AS1 fragment and mutant SATB2-AS1 fragment were inserted downstream of the luciferase reporter vector pmirGLO (Promega Corporation, Madison, WI, USA) to generate pmirGLO-SATB2-AS1-WT and pmirGLOSATB2-AS1-MUT, respectively. HEK293T cells were co-transfected with pmirGLO-SATB2-AS1-WT or pmirGLO-SATB2-AS1-MUT and negative control mimics (NC mimics) or miR-299-3p mimics using Lipofectamine 2000 (Invitrogen). At $48 \mathrm{~h}$ after transfection, luciferase activity was determined using the dual-luciferase reporter assay system (Promega). The firefly luciferase activity was normalized against Renilla luciferase activity.

\section{RNA immunoprecipitation (RIP) assays}

RIP experiments were carried out using the commercially available EZ-Magna RIP Kit (Millipore, Billerica, MA, USA) according to the manufacturer's protocol. RIP buffer containing magnetic beads conjugated with anti-Ago2 antibody or IgG (control) was utilized to obtain the co-precipitants in the cell lysis buffer. Following proteinase $\mathrm{K}$ diges- 
tion, the isolated RNAs were subjected to qRT-PCR analysis.

\section{Statistical analysis}

SPSS 20.0 software (IBM Corp., USA) was used for statistical analysis. All the experiments were conducted in triplicate. Data were presented as mean \pm standard deviation. Two-group comparison was performed using Student's t-test. Multiple group comparison was performed using oneway ANOVA. Survival rate was calculated by the log-rank test and Kaplan-Meier survival analysis. A $p$-value of less than 0.05 was considered as statistically significant.

\section{Results}

\section{SATB2-AS1 up-regulation was observed in NSCLC tissues and cell lines}

To investigate the role of SATB2-AS1 in NSCLC, we initially measured its expression levels in cancerous tissues and matched para-cancerous tissues from 60 patients. As shown in Figure $1 \mathrm{~A}$, the expression of SATB2-AS1 was significantly higher in NSCLC tissues than in normal non-cancerous tissues $(p<0.01)$. Subsequently, we evaluated the expression patterns of SATB2-AS1 in normal plasma cells and four NSCLC cell lines using qRTPCR analysis. As presented in Figure 1 B, SATB2AS1 expression level was significantly elevated in NSCLC cell lines compared with that in the normal human bronchial epithelial cell line BEAS-2B ( $p<$ 0.01 ), which was consistent with the findings in clinical tissue samples. To analyze the relationship between SATB2-AS1 expression levels and survival time, patients were divided into two groups, a high SATB2-AS1 expression group and a low SATB2-AS1 expression group, based on the average value of its expression levels. As is evident from Kaplan-Meier survival analysis, a significantly shorter overall survival was observed in patients with high SATB2-AS1 expression than in those with low SATB2-AS1 expression (mean survival time, 615 days vs 1161 days, $p<0.01$, Figure 1 C). Together, our results suggest that SATB2-AS1 may have a critical role in the development of NSCLC.

\section{SATB2-AS1 facilitates proliferation and suppresses apoptosis of NSCLC cells}

To explore the biological role of SATB2-AS1 in NSCLC, gain- and loss-of-function studies were carried out in A549 cells with the lowest endogenous

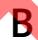

A

B

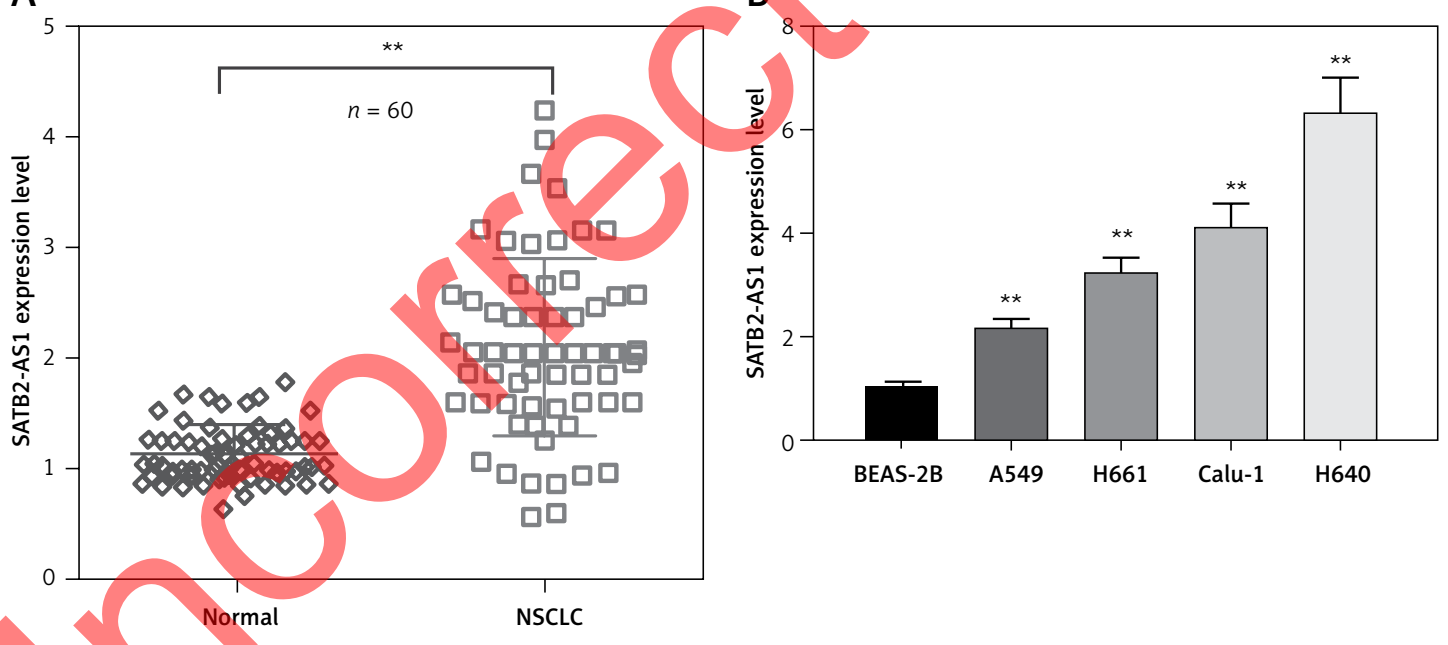

Figure 1. SATB2-AS1 expression is significantly increased in NSCLC tissues and cell lines. A - RT-PCR analysis was used to analyze the expression of SATB2-AS1 in tumorous tissue samples and adjacent normal tissue samples from 60 NSCLC patients. B - RT-PCR analysis was applied to evaluate the expression pattern of SATB-AS1 in the normal human bronchial epithelial cell line BEAS-2B and four NSCLC cell lines. $C$ - The relationship between SATB-AS1 expression and overall survival of NSCLC patients was assessed using the Kaplan-Meier survival curve method and the log-rank test

${ }^{* *} p<0.01$; SATB2-AS1 - the anti-sense transcript of special AT-rich sequence-binding protein 2, NSCLC - nonsmall cell lung cancer. 
SATB2-AS1 expression and $\mathrm{H} 460$ cells with the highest endogenous SATB2-AS1 expression, respectively. NSCLC cells were transfected with SATB2-AS1 expression vectors or SATB2-AS1-specific shRNA (shSATB2-AS1) to achieve regulation of its expression. As displayed in Figure $2 \mathrm{~A}$, the SATB2-AS1 expression level was notably higher in the SATB2-AS1 over-expression group than that in the empty control group $(p<0.01)$; the SATB2-AS1 expression level was considerably lower in the SATB2-AS1 knockdown group in comparison with that in the negative control group ( $p<0.01$ ). The effects of SATB2-AS1 on NSCLC cell proliferation, cell cycle and apoptosis were then determined via MTT assays and flow cytometry. As presented in Figure 2 B, SATB2-AS1 over-expression significantly increased the proliferation of A549 cells, whereas SATB2-AS1 knockdown resulted in a notable decrease in $\mathrm{H} 460$ cell proliferation $(p<0.01)$. As shown in Figure $2 \mathrm{C}$, transfection of SATB2-AS1 expression vectors accelerated A549 cell cycle progres- sion compared with the control group, while transfection of sh-SATB2-AS1 induced cell cycle arrest in $\mathrm{H} 460$ cells in comparison with negative control treatment. Furthermore, the apoptosis rate of A549 cells was $50.59 \%$ lower in the SATB2-AS1 over-expression group than the control group $(p<0.01)$, whereas the apoptosis rate of $\mathrm{H} 460$ cells was $62.51 \%$ higher in the SATB2-AS1 knockdown group than the negative control group ( $p<0.01$, Figure $2 \mathrm{D}$ ).

In addition, we evaluated the effects of SATB2AS1 on proliferation-related proteins ( $p-R b$, CCND1, CDK4/6 and C-myc) and apoptosis-associated proteins (Bax, cleaved caspase-3, Bcl2 and Mcl-1) using Western blotting. As displayed in Figure $2 \mathrm{E}$, higher expression levels of $\mathrm{p}-\mathrm{Rb}, \mathrm{CCND} 1$, $\mathrm{CDK} 4 / 6, \mathrm{C}-\mathrm{myc}, \mathrm{Bcl} 2$ and $\mathrm{Mcl}-1$ and lower expression levels of Bax and cleaved caspase- 3 were observed in the over-expression group than in the empty control group $(p<0.01)$, whereas SATB2AS1 depletion significantly reduced the expres-

A
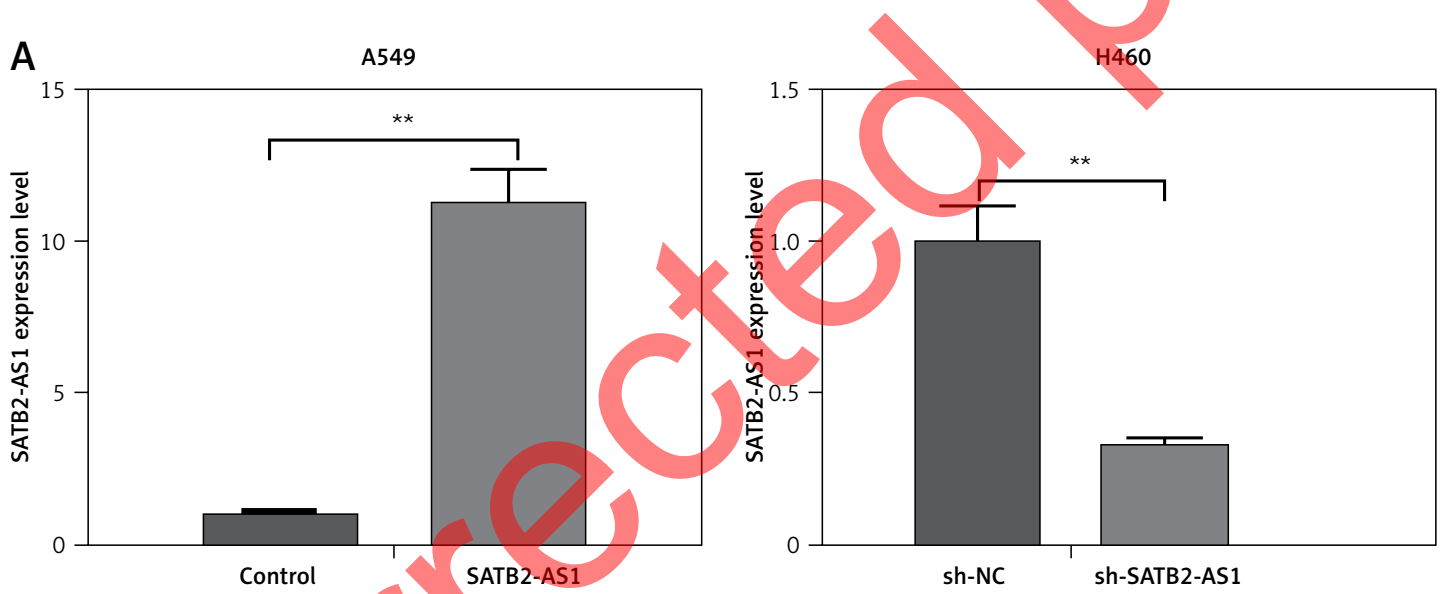

B

A549
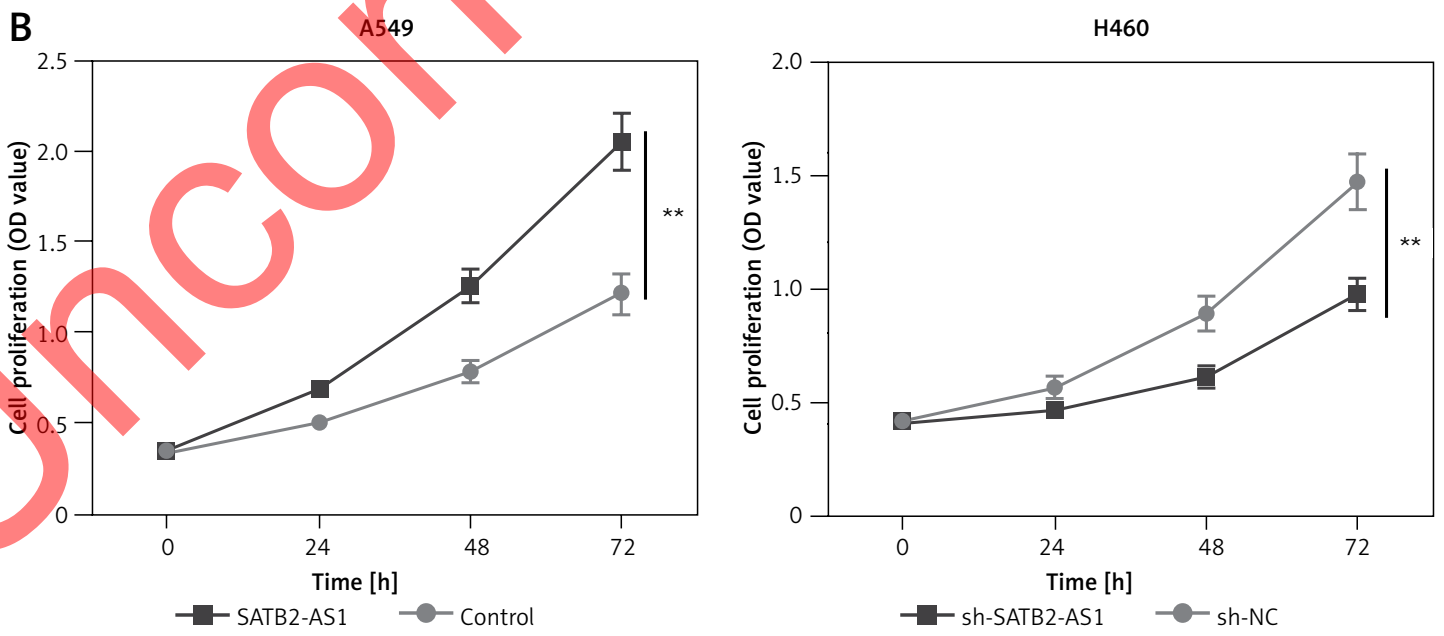

Figure 2. SATB2-AS1 facilitates proliferation and suppresses apoptosis of NSCLC cells. A - NSCLC cells were transfected with SATB2-AS1 over-expression vectors and sh-SATB2-AS1 to modulate SATB2-AS1 expression; SATB2-AS1 expression levels were measured using RT-PCR analysis at $48 \mathrm{~h}$ after transfection. $\mathrm{B}-\mathrm{MTT}$ assays were used to evaluate cell proliferation after transfection with empty vectors, SATB2-AS1 expression vectors, sh-NC or sh-SATB2-AS1 ${ }^{* *} p<0.01$; SATB2-AS1 - the anti-sense transcript of special AT-rich sequence-binding protein 2, NSCLC - non-small cell lung cancer, sh-NC - negative control short hairpin RNA, sh-SATB2-AS1 - short hairpin RNA specifically targeting SATB2-AS1, $p$-Rbphosphorylated retinoblastoma protein, CCND1 - cyclin D1, CDK4/6 - cyclin-dependent kinase 4/6, Bcl2 - B cell lymphoma 2; Bax-Bcl2-associated X protein, Mcl1-myeloid cell leukemia 1 protein. 
$\mathrm{C}$
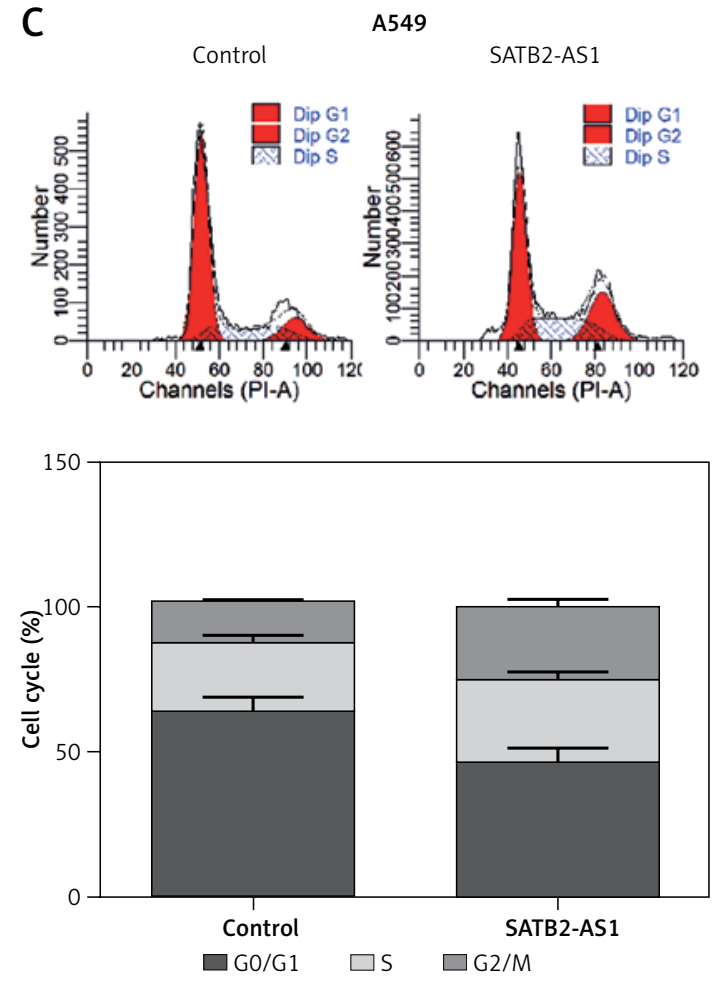

sh-NC
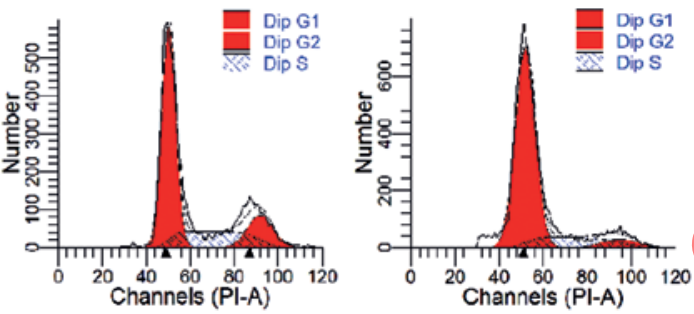

D

A549
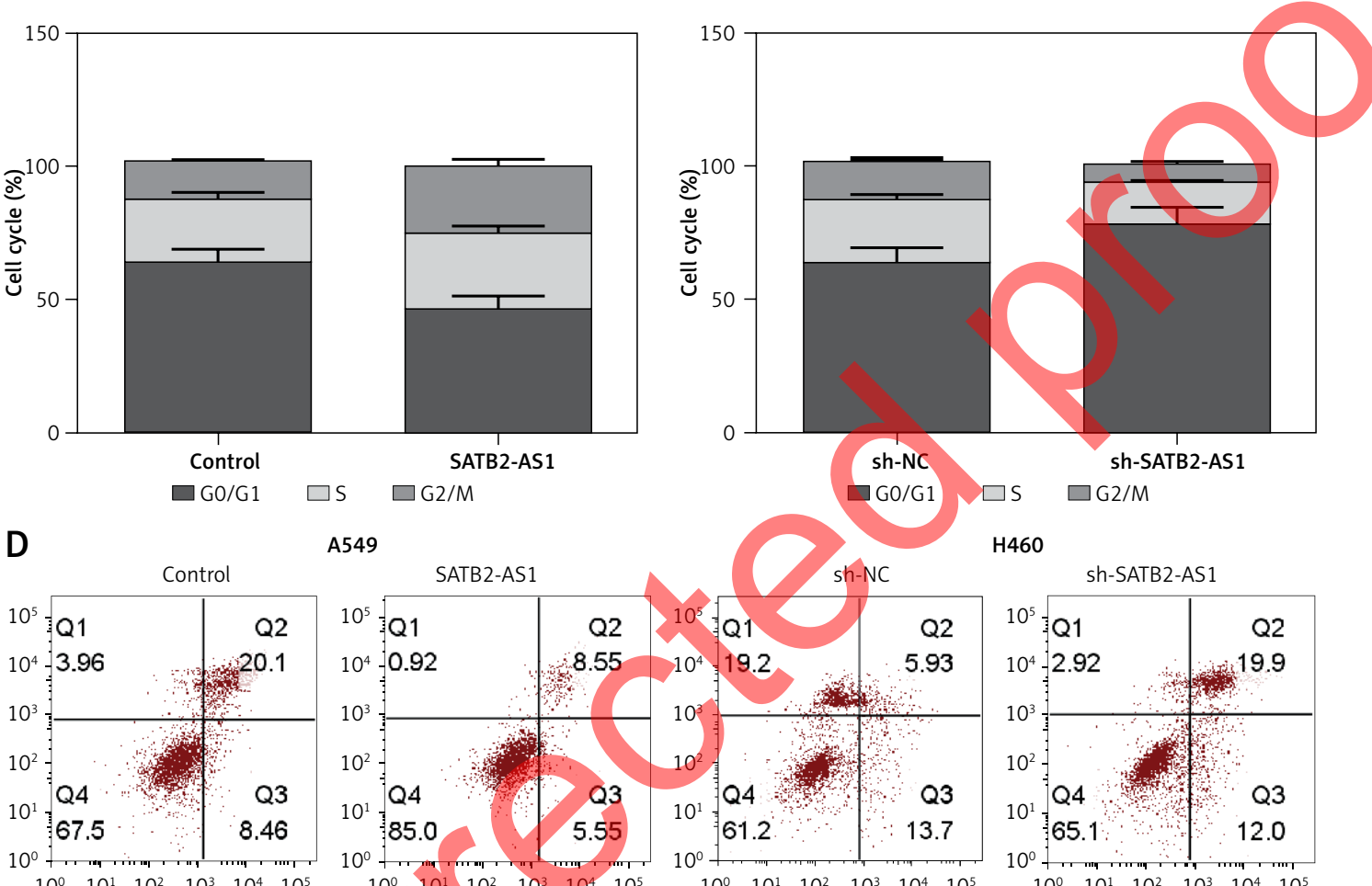

SATB2-AS1

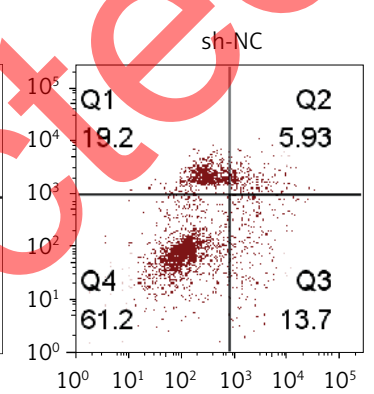

$\mathrm{H} 460$
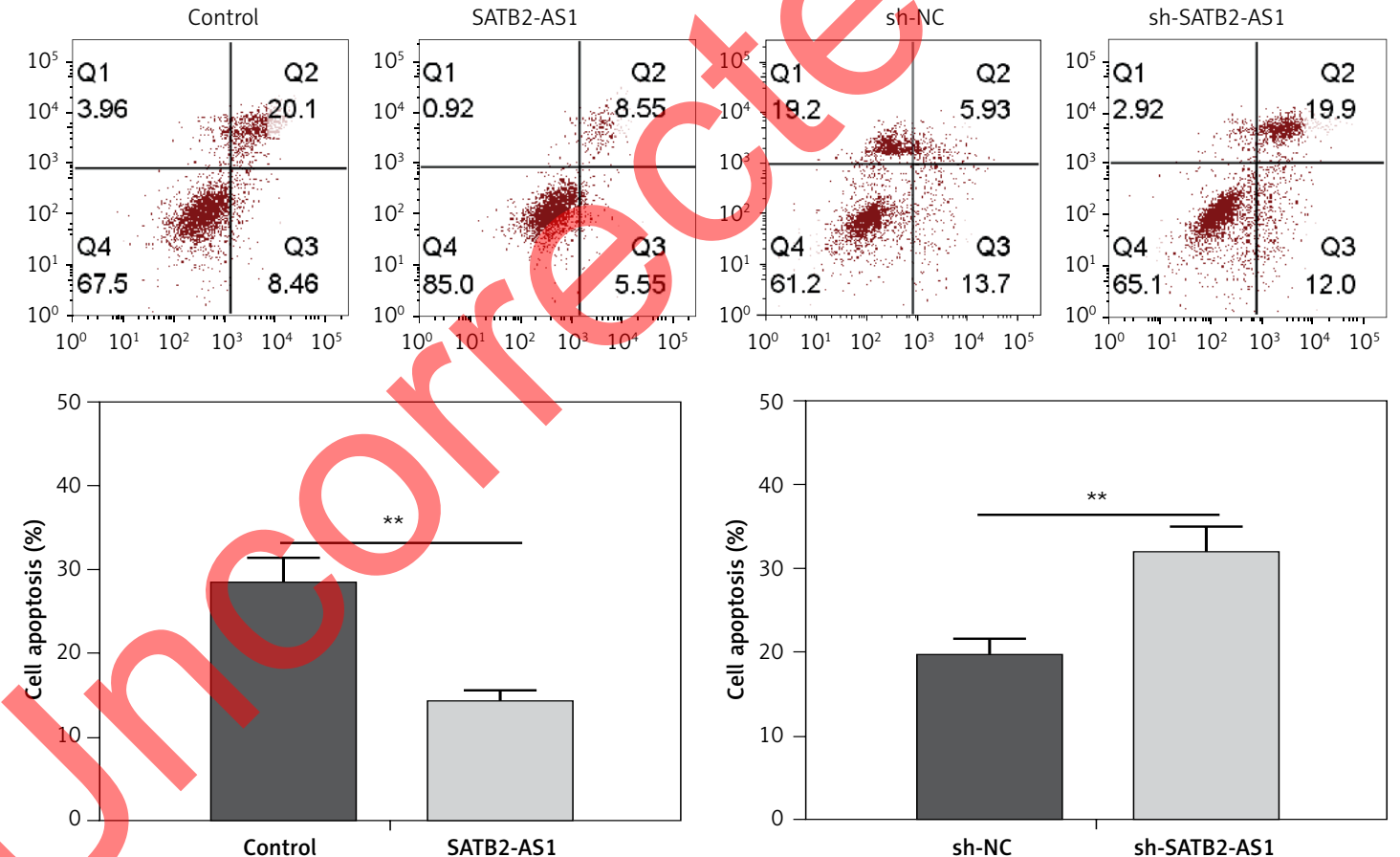

Figure 2. Cont. C - Cell cycle progression was determined using flow cytometry after transfection with empty vectors, SATB-AS1 expression vectors, sh-NC or sh-SATB2-AS1. D - Apoptosis of NSCLC cells were examined using flow cytometry after transfection with empty vectors, SATB2-AS1 expression vectors, sh-NC or sh-SATB2-AS1

${ }^{* *} p<0.01$; SATB2-AS1 - the anti-sense transcript of special AT-rich sequence-binding protein 2, NSCLC - non-small cell lung cancer, sh-NC - negative control short hairpin RNA, sh-SATB2-AS1 - short hairpin RNA specifically targeting SATB2-AS1, $p$-Rb phosphorylated retinoblastoma protein, CCND1 - cyclin D1, CDK4/6 - cyclin-dependent kinase 4/6, Bcl2 - B cell lymphoma 2; Bax-Bcl2-associated X protein, Mcl1-myeloid cell leukemia 1 protein. 
$E$

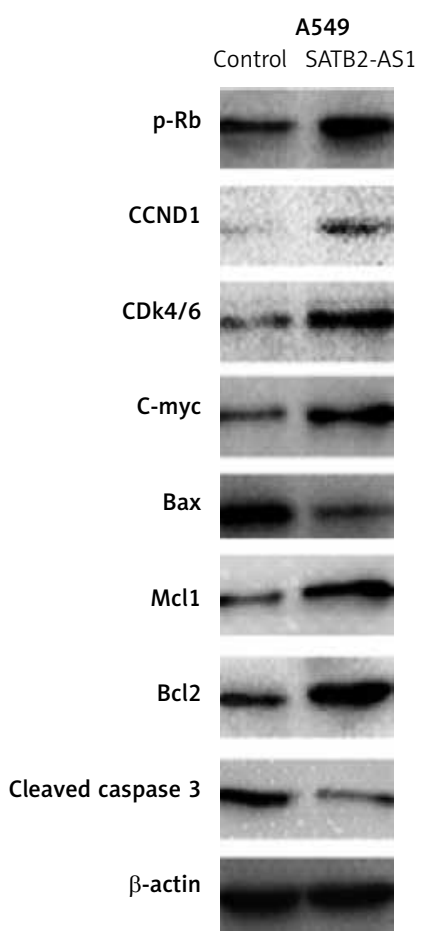

$\mathrm{H} 460$

sh-NC sh-SATB2-AS1

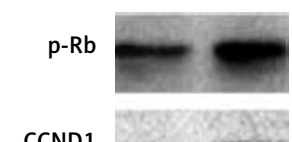

CCND1

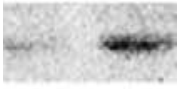

$\mathrm{CDk} 4 / 6$

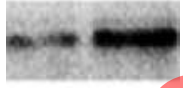

C-myc
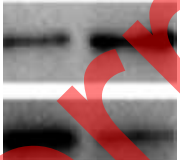

$\mathrm{Mcl} 1$

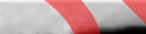

$\mathrm{Mcl}$

$\mathrm{Bcl} 2$

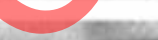

Cleaved caspase 3

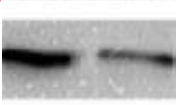

$\beta$-actin

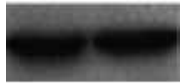

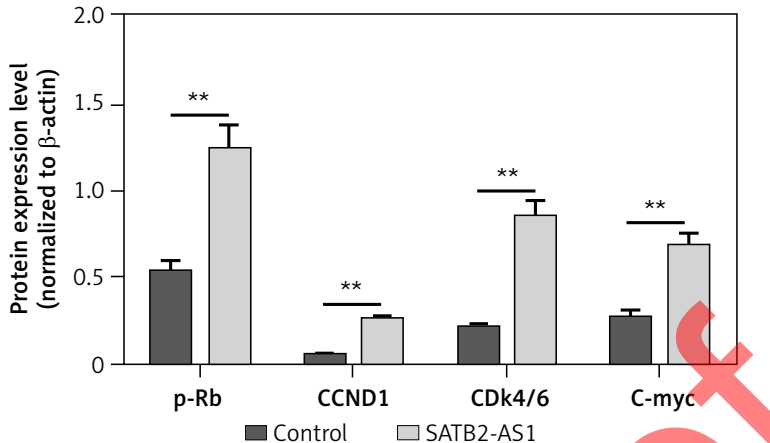

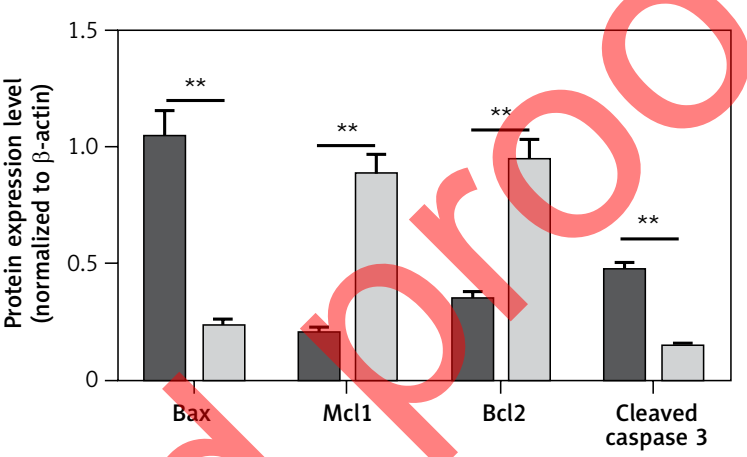

$\square$ SATB2-AS1
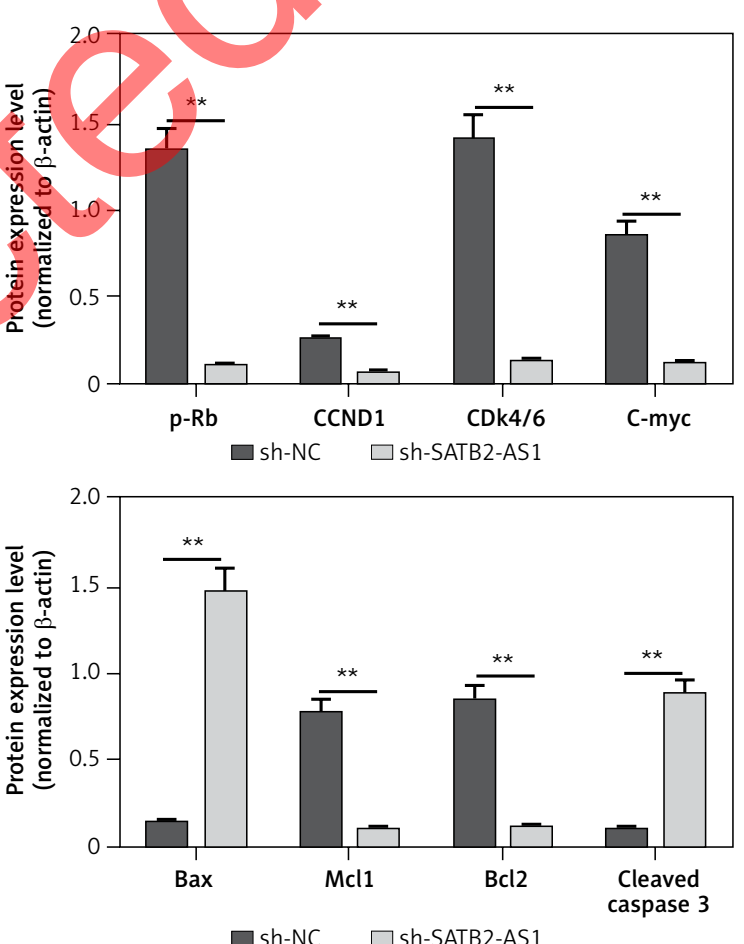

Figure 2. Cont. E - Expression levels of proliferation-related proteins ( $\mathrm{p}-\mathrm{Rb}, \mathrm{CCND} 1, \mathrm{CDK} 4 / 6$ and C-myc) and apoptosis-related proteins (Bax, Mcl1, Bcl2 and cleaved caspase 3) were analyzed using Western blotting in NSCLC cells transfected with empty vectors, SATB2-AS1 expression vectors, sh-NC or sh-SATB2-AS1

${ }^{* *} p<0.01$; SATB2-AS1 - the anti-sense transcript of special AT-rich sequence-binding protein 2, NSCLC - non-small cell lung cancer, sh-NC - negative control short hairpin RNA, sh-SATB2-AS1 - short hairpin RNA specifically targeting SATB2-AS1, $p$-Rb phosphorylated retinoblastoma protein, CCND1 - cyclin D1, CDK4/6 - cyclin-dependent kinase 4/6, Bcl2 - B cell lymphoma 2; Bax-Bcl2-associated X protein, Mcl1-myeloid cell leukemia 1 protein. 
sion levels of $\mathrm{p}-\mathrm{Rb}, \mathrm{CCND} 1, \mathrm{CDK} 4 / 6, \mathrm{C}-\mathrm{myc}, \mathrm{Bcl} 2$ and $\mathrm{Mcl}-1$ and elevated the expression levels of Bax and cleaved caspase- 3 ( $p<0.01)$. Collectively, these data indicate that SATB2-AS1 promotes NSCLC cell proliferation, accelerates cell cycle progression and inhibits apoptosis.

\section{SATB2-AS1 ablation inhibits tumorigenicity of NSCLC cells in mouse xenograft models}

To investigate the effects of SATB2-AS1 on tumorigenicity of NSCLC cells in vivo, tumor transplan- tation models were established via subcutaneous injection of sh-NC- or sh-SATB2-AS1-treated H460 cells in the flanks of the nude mice. As shown in Figure $3 \mathrm{~A}$, tumors formed by sh-SATB2-AS1-treated $\mathrm{H} 460$ cells grew more slowly than those formed by sh-NC-treated $\mathrm{H} 460$ cells $(p<0.01$ ); moreover, tumors collected from the sh-SATB2-AS1 group had a much lower weight than those harvested from the sh-NC group $(p<0.01)$. Furthermore, tumors in the SATB2-AS1 ablation group displayed lower Ki67 expression levels than those in the
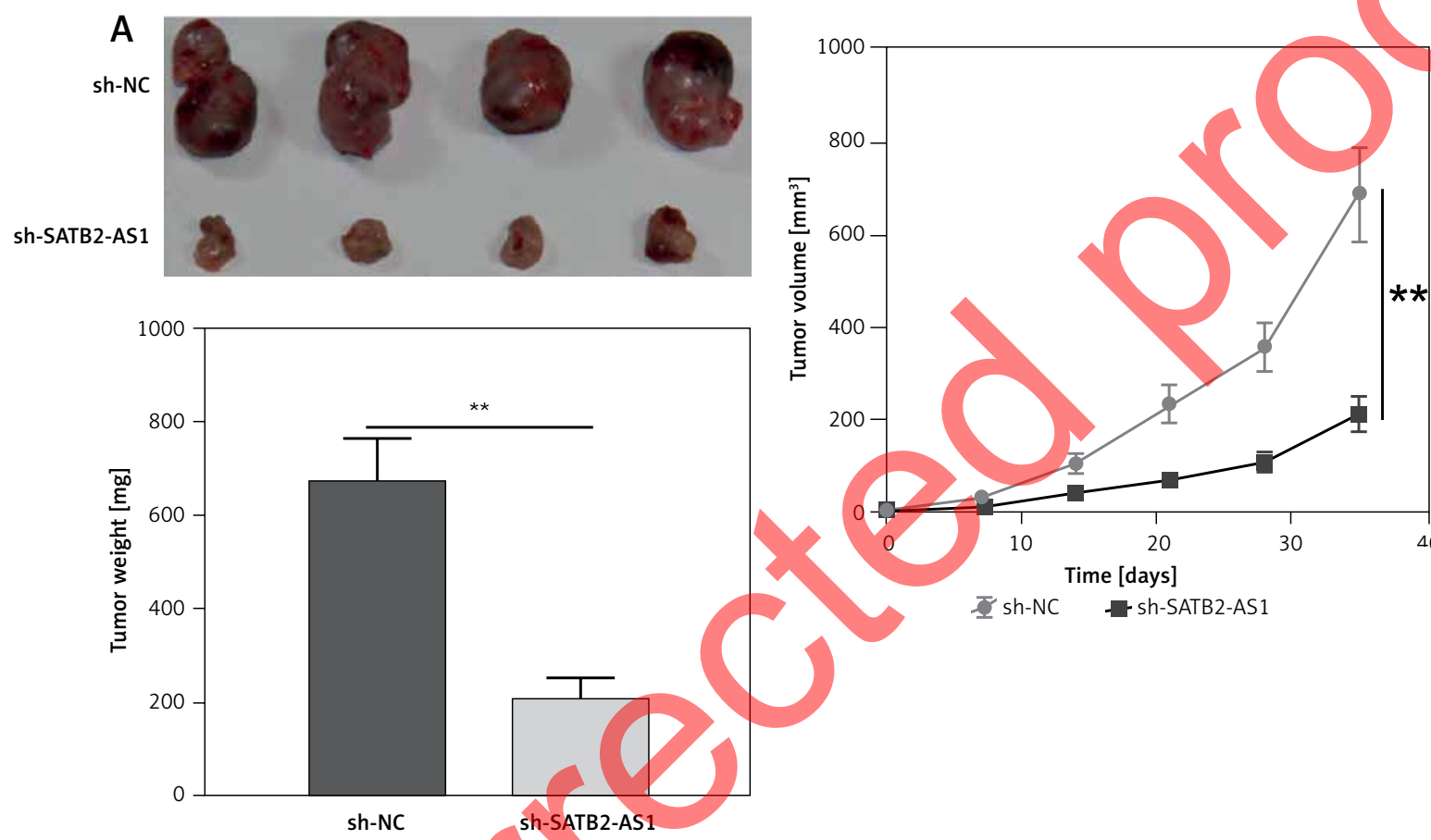

B Ki67
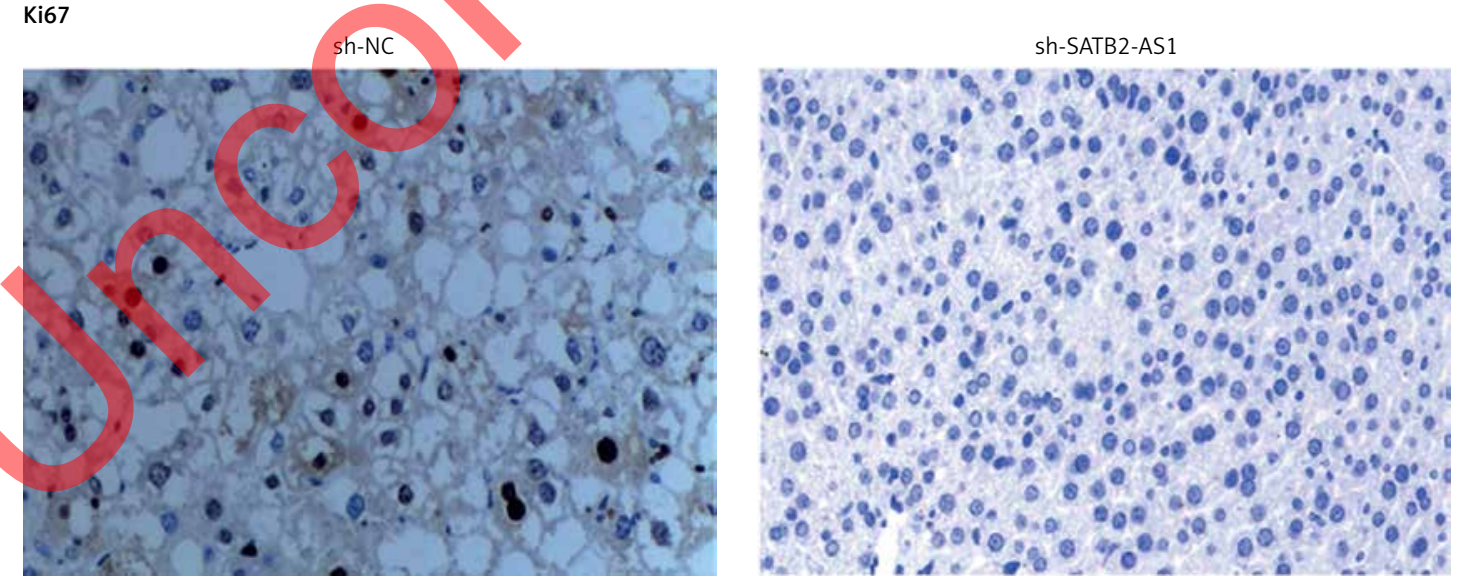

Figure 3. SATB2-AS1 ablation inhibits tumorigenicity of NSCLC cells in mouse xenograft models. A - sh-NC- or sh-SATB2-AS1-treated $\mathrm{H} 460$ cells were inoculated into the flanks of the nude mice. The tumor growth curve was plotted based on measurement data taken every 7 days; mice were sacrificed at post-transplantation day 35 , and tumors were removed and weighed. B - Immunohistochemical staining was conducted to detect Ki67-positive cells in the collected tumors formed by sh-NC- or sh-SATB2-AS1-treated H460 cells

${ }^{* *} p<0.01$; SATB2-AS1 - the anti-sense transcript of special AT-rich sequence-binding protein 2, NSCLC - non-small cell lung cancer, sh-NC - negative control short hairpin RNA, sh-SATB2-AS1 - short hairpin specifically targeting SATB2-AS1, TUNEL - terminal deoxynucleotidyl transferase mediated nick end labeling. 
C

DAPI

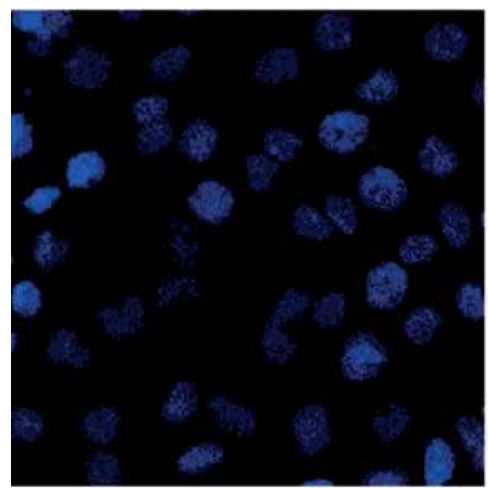

sh-NC

sh-SATB2-AS1

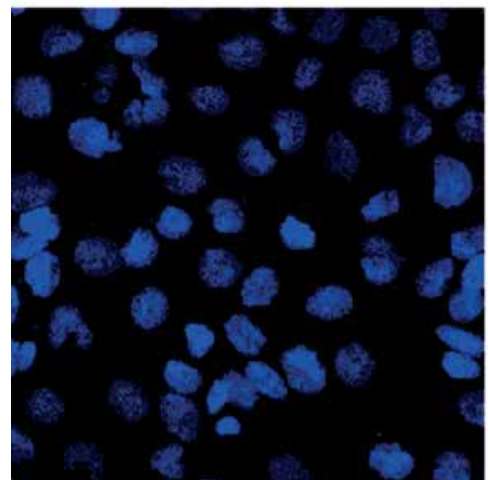

TUNEL
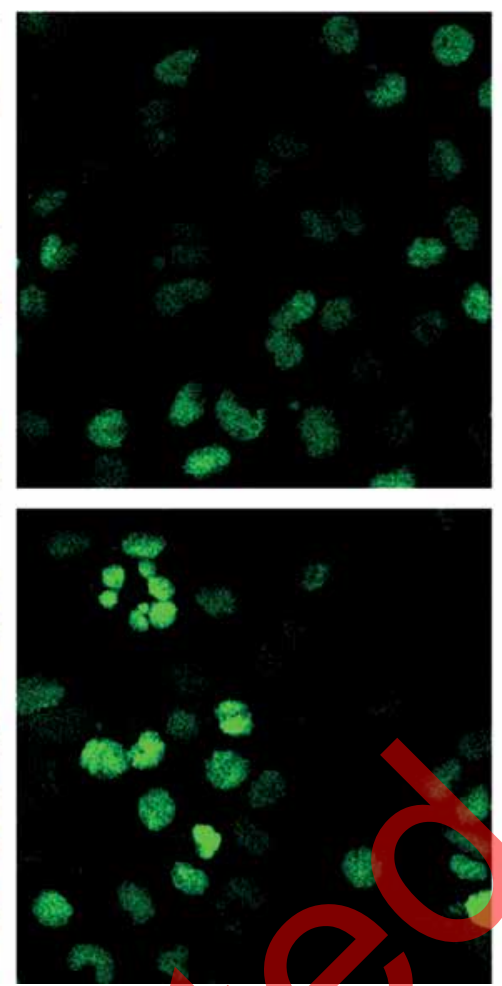

Merge
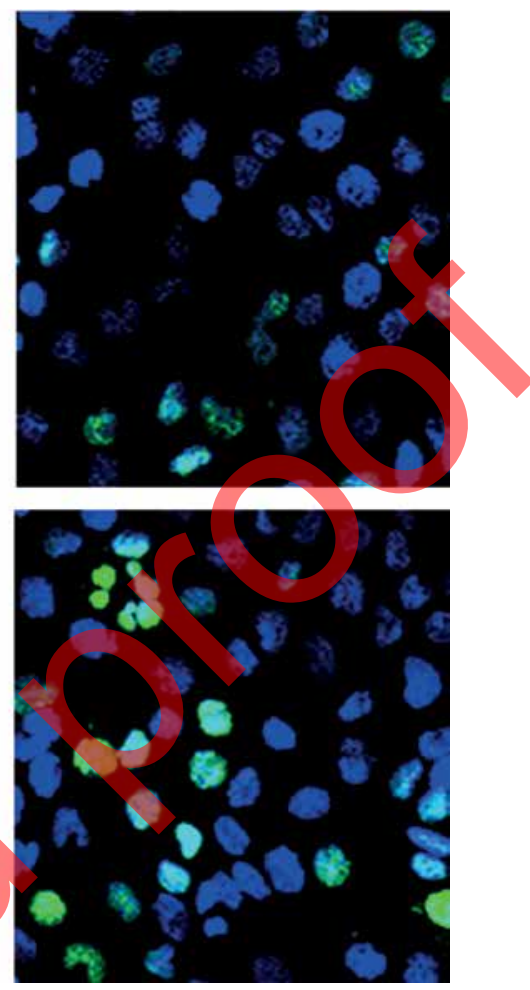

Figure 3. Cont. C - DAPI/TUNEL double staining was used to examine apoptotic cells in the harvested tumors ${ }^{* *} p<0.01$; SATB2-AS1 - the anti-sense transcript of special AT-rich sequence-binding protein 2, NSCLC - non-small cell lung cancer, sh-NC - negative control short hairpin RNA, sh-SATB2-AS1 - short hairpin specifically targeting SATB2-AS1, TUNEL - terminal deoxynucleotidyl transferase mediated nick end labeling.

negative control group (Figure 3 B). Additionally, DAPI/TUNEL double staining showed that more apoptotic cells were observed in the SATB2-AS1 knockdown group than in the negative control group (Figure $3 \mathrm{C}$ ). To sum up, these data imply that SATB2-AS1 depletion represses tumorigenicity of NSCLC cells invivo.

\section{SATB2-AS1 serves as a molecular sponge of miR-299-3p}

Accumulating data have revealed that crosstalk is common between IncRNAs and miRNAs and that IncRNAs could act as molecular decoys of miRNAs to execute their functions. To elucidate the possible molecular mechanisms by which SATB2-AS1 contributes to proliferation, cell cycle progression and survival of NSCLC cells, we next conducted further mechanistic studies. Herein, we carried out bio-informatics prediction using the starBase v3.0 online database. As shown in Figure $4 \mathrm{~A}$, there was a putative complementary binding site for SATB2-AS1 in miR-299-3p. Afterwards, RIP analysis was performed to determine whether SATB2-AS1 interacted with miR-299-3p. As presented in Figure 4 B, more miR-299-3p was enriched in the SATB2-AS1 over-expression group than in the control group. Furthermore, dual luciferase reporter assays demonstrated that miR299-3p mimics led to a significant decrease in the luciferase activity of the wild-type SATB2-AS1 reporter vectors compared with negative control treatment, whereas there was no significant difference in the luciferase activity of the mutant SATB2-AS1 reporter vectors between the NC mimic group and the miR-299-3p mimic group (Figure $4 \mathrm{C}$ ).

Additionally, the effects of SATB2-AS1 on miR299-3p expression were assessed via qRT-PCR analysis. As displayed in Figure 4 D, SATB2-AS1 over-expression significantly reduced the expression levels of miR-299-3p in A549 cells in comparison with the control group $(p<0.01)$, while SATB2AS1 depletion significantly elevated the expression levels of miR-299-3p in H460 cells compared with the negative control group ( $p<0.01)$. Notably, miR299-3p expression was significantly decreased in NSCLC tissues compared with adjacent normal tissues from healthy volunteers ( $p<0.01$, Figure $4 \mathrm{E}$ ). Furthermore, an inverse correlation was observed between SATB2-AS1 expression and miR-299-3p expression in NSCLC tissues (Figure $4 \mathrm{~F}$ ). Together, these data suggest that SATB2-AS1 functions as a molecular sponge of miR-299-3p. 
A $\begin{array}{ll}\text { SATB2-AS1 } & \text { 5'AAACCCCUUCCCACAU-3' } \\ \text { miR-299-3p } & \text { 3'-AAAUGGUAGGGUGUA-5' }\end{array}$

C

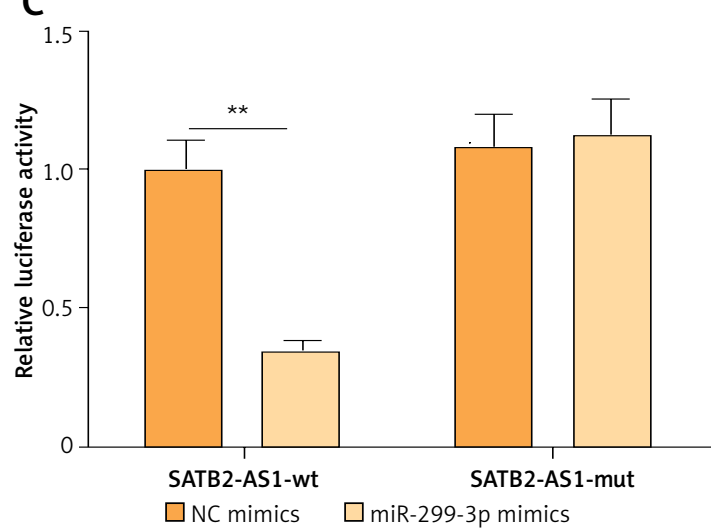

D

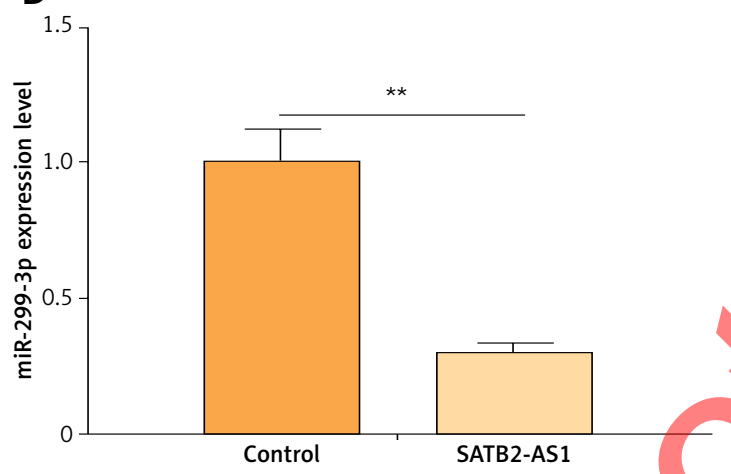

$\mathrm{E}$

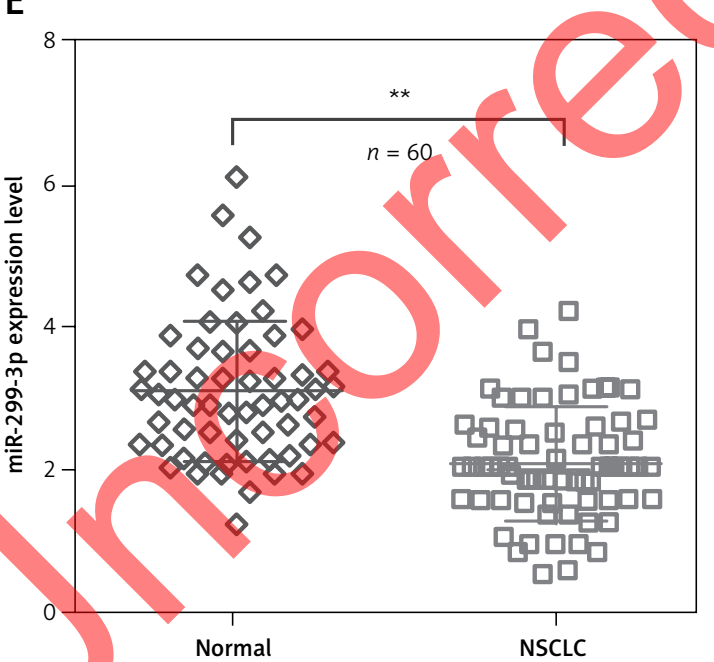

B

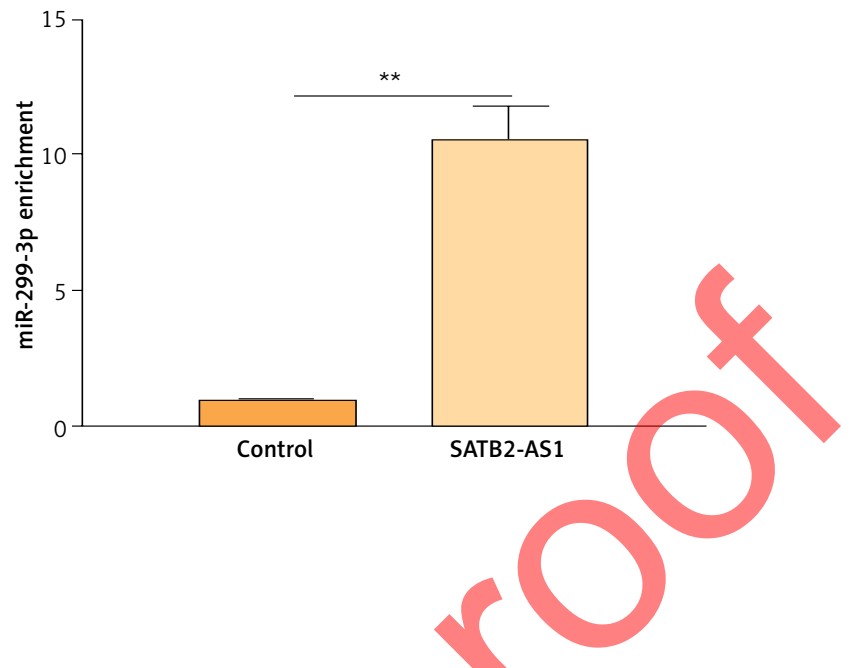

Figure 4. SATB2-AS1 serves as a molecular sponge of miR-299-3p. A - A potential binding site of SATB2-AS1 in miR-299-3p was predicted using the starBase v3.0 online algorithms. B - RNA immuno-precipitation assays were applied to validate the interaction between SATB2-AS1 and miR-299-3p in HEK293T cells. C - Dual luciferase reporter assays were performed to verify whether SATB2-AS1 could directly bind to miR-299-3p in HEK293T cells. D - miR-299-3p expression levels were determined in NSCLC cells using RT-PCR analysis after transfection with empty vectors, SATB2-AS1 expression vectors, sh-NC or sh-SATB2-AS1. E - miR-299-3p expression levels were examined using RT-PCR analysis in NSCLC tissues and adjacent normal tissues of 60 patients. F - Relationship between SATB2-AS1 expression and miR-299-3p expression in NSCLC tissues was assessed using Spearman correlation analysis

${ }^{* *} p<0.01$; SATB2-AS1 - the anti-sense transcript of special AT-rich sequence-binding protein 2, NSCLC - multiple myeloma, sh-NC - negative control short hairpin RNA, sh-SATB2-AS1 - short hairpin RNA specifically targeting SATB2-AS1, SATB2-AS1-wt - wild-type SATB2-AS1, SATB2-AS1-mut - mutant SATB2-AS1, NC mimics - negative control mimics. 
A

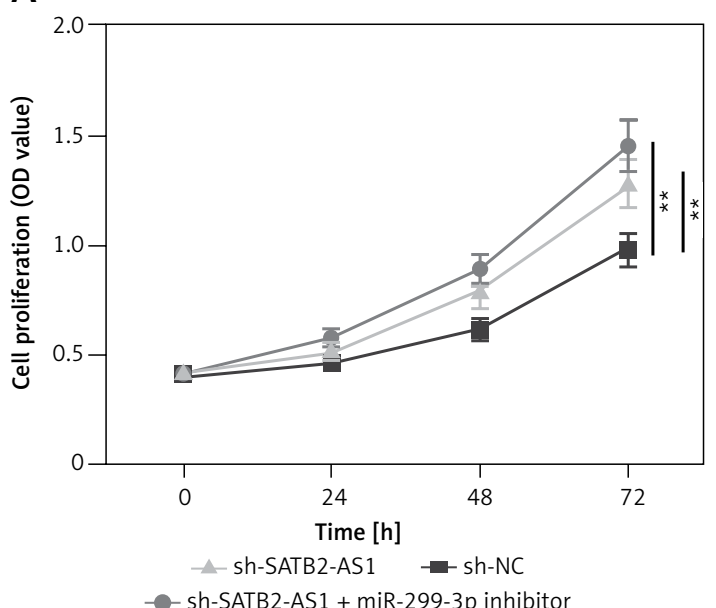

B1

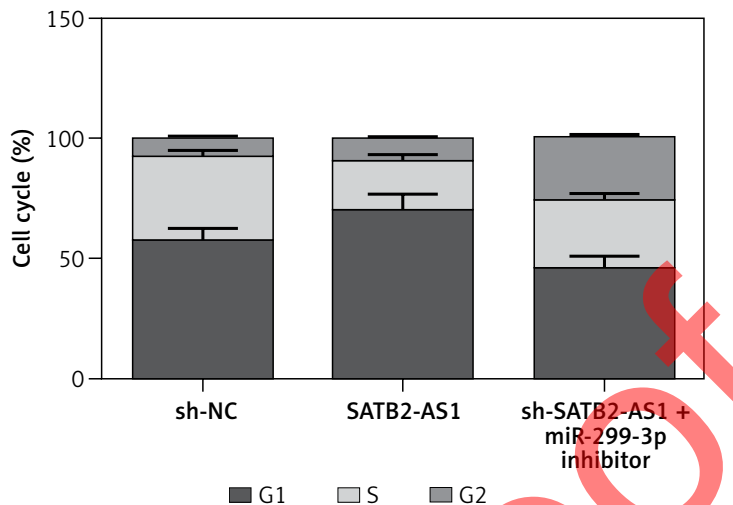

B2

sh-SATB2-AS1

miR-299-3p inhibitor -
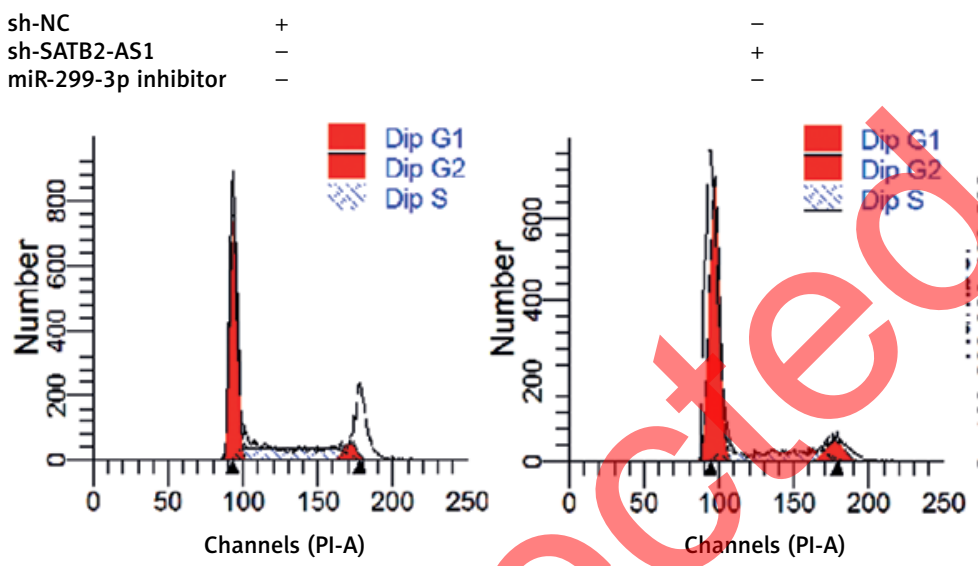

C

sh-NC

Sh-SATB2-AS1

miR-299-3p inhibitor
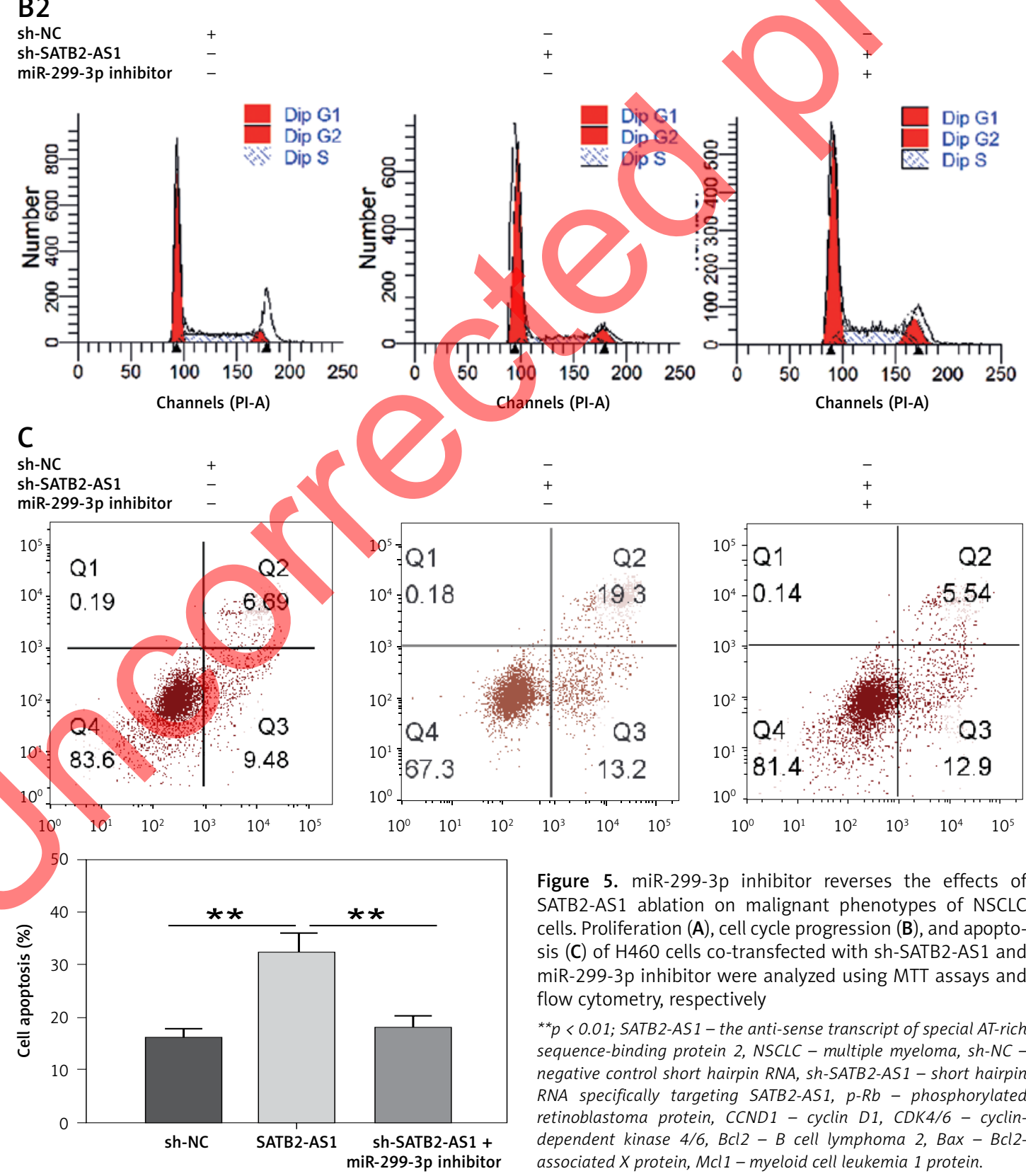

Figure 5. miR-299-3p inhibitor reverses the effects of SATB2-AS1 ablation on malignant phenotypes of NSCLC cells. Proliferation (A), cell cycle progression (B), and apoptosis (C) of H460 cells co-transfected with sh-SATB2-AS1 and miR-299-3p inhibitor were analyzed using MTT assays and flow cytometry, respectively

${ }^{* *} p<0.01$; SATB2-AS1 - the anti-sense transcript of special AT-rich sequence-binding protein 2, NSCLC - multiple myeloma, sh-NC negative control short hairpin RNA, sh-SATB2-AS1 - short hairpin RNA specifically targeting SATB2-AS1, $p-R b$ - phosphorylated retinoblastoma protein, CCND1 - cyclin D1, CDK4/6 - cyclindependent kinase 4/6, BCl2 - B cell lymphoma 2, Bax - Bcl2associated $X$ protein, Mcl 1 - myeloid cell leukemia 1 protein. 
D sh-NC Sh-SATB2-AS1 miR-299-3p inhibitor

$\mathrm{p}-\mathrm{Rb}$

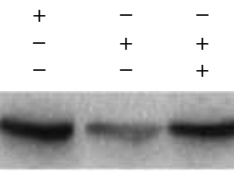

CCND1

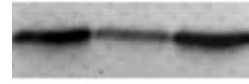

$\mathrm{CDk} 4 / 6$

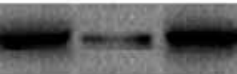

C-myc

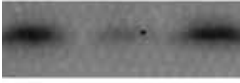

Bax

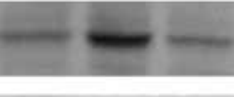

Mcl1

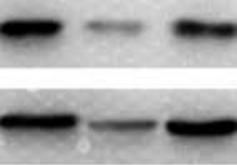

Cleaved caspase 3

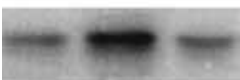

$\beta$-actin

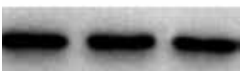

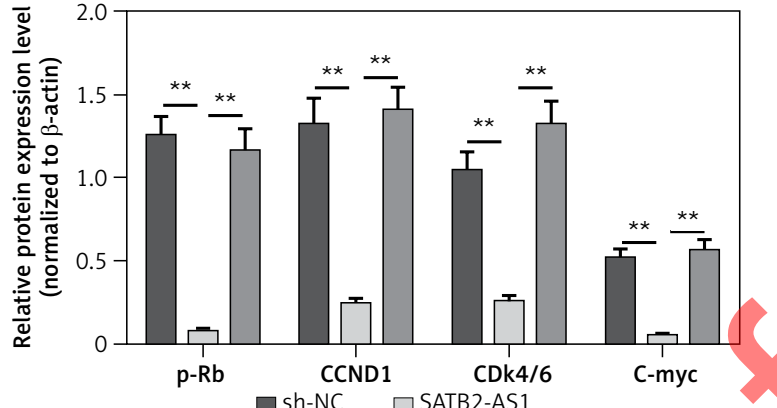

sh-SATB2-AS1 + miR-299-3p inhibitor

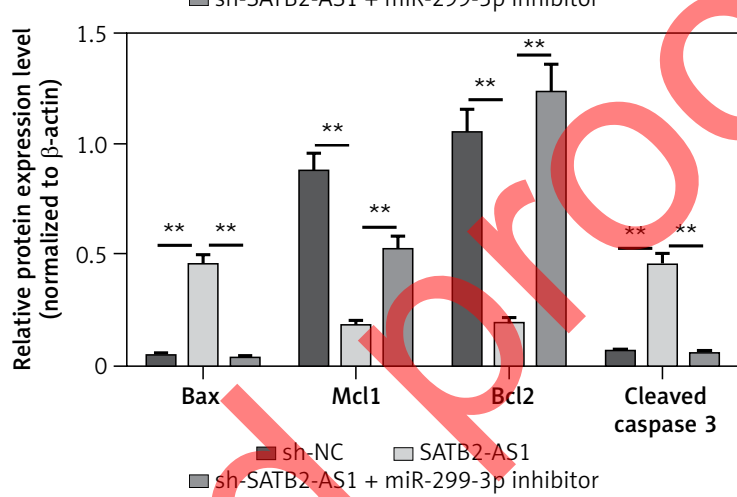

Figure 5. Cont. D - Expression levels of proliferation-related proteins ( $p$-Rb, CCND1, CDK4/6 and C-myc) and apoptosis-related proteins (Bax, Mcl1, Bcl2 and cleaved caspase 3) were determined using Western blotting in H460 cells co-transfected with sh-SATB2-AS1 and miR-299-3p

${ }^{* *} p<0.01$; SATB2-AS1 - the anti-sense transcript of special AT-rich sequence-binding protein 2, NSCLC-multiple myeloma, sh-NC - negative control short hairpin RNA, sh-SATB2-AS1 - short hairpin RNA specifically targeting SATB2-AS1, p-Rb-phosphorylated retinoblastoma protein, CCND1 - cyclin D1, CDK4/6 - cyclin-dependent kinase 4/6, BCl2 - B cell lymphoma 2, Bax - Bcl2-

associated X protein, Mcl1-myeloid cell leukemia 1 prote

miR-299-3p inhibitor reverses the effects of SATB2-AS1 knockdown on malignant phenotypes of NSCLC cells

To determine the functional relationship between SATB2-AS1 and miR-299-3p, we analyzed the effects of miR-299-3p inhibitor on malignant phenotypes of sh-SATB2-AS1-treated $\mathrm{H} 460$ cells. As exhibited in Figures 5 A, B, the inhibitory effects of SATB2-AS1 knockdown on H460 cell proliferation and cell cycle progression were significantly alleviated by miR-299-3p inhibitor. Moreover, miR299-3p inhibitor reversed the apoptosis-promoting effect induced by SATB2-AS1 ablation in H460 cells (Figure 5 C). Remarkably, miR-299-3p inhibitor also attenuated sh-SATB2-AS1-induced facilitating effects on Bax and cleaved caspase- 3 expression and suppressing effects on p-Rb, CCND1, CDK4/6, C-myc, Mcl-1 and Bcl2 expression in $\mathrm{H} 460$ cells (Figure $5 \mathrm{D}$ ). Taken together, these results imply that SATB2-AS1 acts as a miR-299-3p sponge to contribute to NSCLC cell proliferation and survival.

\section{Discussion}

In recent decades, constant efforts in cellular and molecular biology have led to explosive discoveries demonstrating that IncRNAs serve as crucial regulators during the initiation and pro- gression of human tumors and other disorders $[33,34]$. It is well accepted that characterization of the de-regulated IncRNAs could advance our comprehensive understanding of cancer biology and treatment. NSCLC, one of the most aggressive human malignancies, is responsible for an increasing frequency of cancer-associated mortality [14, 15]. Accumulating data indicate that IncRNAs are involved in NSCLC development and that the dysregulation of IncRNAs contributes to malignant progression of NSCLC. Zhu et al. demonstrated that IncRNA PART1 was significantly up-regulated in NSCLC and that IncRNA PART1 facilitated NSCLC development via the JAK-STAT signaling pathway [11]. Wu and his colleagues reported the involvement of InCRNA DLEU2 in NSCLC, and they found that InCRNA DLEU2 accelerated the tumorigenesis and invasion by sponging miR-30-5p [12]. Huang's research group discovered that IncRNA AFAP1-AS1 was highly expressed in NSCLC and that InCRNA PART1 promoted tumor cell proliferation and chemotherapy resistance through regulation of RRM2 [13]. It is widely accepted that characterization of novel tumor-associated IncRNAs could contribute to solving the mystery of NSCLC development.

In the past few years, SATB2-AS1 has been demonstrated to serve as a pivotal regulator in human carcinogenesis and development. Liu and 
his research group reported that SATB2-AS1 was over-expressed in osteosarcoma tissues and cell lines and that SATB2-AS1 facilitated cancer cell proliferation and growth [27]. Wang et al. determined the expression pattern of SATB2-AS1 in colorectal carcinoma; they found that SATB2-AS1 expression was significantly decreased in colorectal carcinoma and that SATB2-AS1 exerted tumor-suppressing functions via inhibiting epithelial-to-mesenchymal transition and aggressiveness [28]. The discrepant results regarding SATB2-AS1 expression in different kinds of human tumors may be attributed to the hypothesis that SATB2AS1 possesses tissue-specific expression patterns, thereby exerting different biological roles in diverse types of human malignancies. Nonetheless, the knowledge concerning the role of SATB2-AS1 in NSCLC is still limited.

In the current study, SATB2-AS1 expression was demonstrated to be significantly elevated in NSCLC tissue samples in comparison with adjacent normal tissue samples from healthy volunteers. Strikingly, increased SATB2-AS1 expression was found to be linked to poor outcome of patients. Based on the above data, we speculated that SATB2-AS1 may act as an oncogene in NSCLC. Gain- and loss-of-function analysis is widely considered as an effective method to investigate the biological role of IncRNAs. Herein, functional investigations showed that over-expression of SATB2AS1 promoted NSCLC cell proliferation, cell cycle progression and survival, whereas knockdown of SATB2-AS1 inhibited NSCLC cell growth in vitro and in vivo. Interestingly, our data also demonstrated that SATB2-AS1 could modulate the expression of proliferation-related proteins and apoptosis-related proteins. It is well documented that increased expression of $\mathrm{p}-\mathrm{Rb}, \mathrm{CCND} 1, \mathrm{CDK} 4 / 6$ and C-myc contributes to uncontrolled cell proliferation [33, 34]. Furthermore, it is widely acknowledged that elevated expression of $\mathrm{MCl}-1$ and $\mathrm{Bcl} 2$ and reduced expression of Bax and cleaved caspase- 3 are intimately related to enhanced cell survival $[35,36]$.

Recently, a large amount of studies have proposed that a crosstalk exists between IncRNAs and miRNAs and that IncRNAs may serve as endogenous molecular sponges of miRNAs to exert their roles $[37,38]$. To better clarify the potential mechanisms underlying the carcinogenic effects of SATB2-AS1 in NSCLC, bio-informatics prediction and relevant validation assays were performed to launch mechanistic studies. In the current study, our results confirmed SATB2-AS1 as a miR-299-3p sponge. Interestingly, rescue assays revealed that miR-299-3p mediated the effects of SATB2-AS1 on NSCLC cell malignant phenotypes and related protein expression. Notably, miR-299-3p has been reported to be significantly down-regulated and act as a tumor suppressor in multiple types of human malignancies, such as cervical cancer [39], renal cell carcinoma [40], thyroid cancer [41], hepatocellular carcinoma [42] and non-small cell lung cancer [43]. Collectively, our results indicate that SATB2-AS1 exerts its oncogenic functions in NSCLC through sponging miR-299-3p.

In summary, this study for the first time demonstrated that SATB2-AS1 expression was significantly elevated in NSCLC tissues and that high SATB2-AS1 expression was associated with poor outcome in patients. Herein, our results revealed that SATB2-AS1 acted as a miR-299-3p sponge to exert carcinogenic effects in NSCLC, including facilitating tumor cell growth, cell cycle progression and survival. Strikingly, we highlighted a novel regulatory mechanism of the SATB2-AS1/miR-299-3p axis in NSCLC development. Collectively, our findings provide new insights into understanding the functional role of SATB2-AS1 in NSCLC, indicating that the SATB2-AS1/miR-299-3p axis represents a candidate therapeutic target for NSCLC.

\section{Conflict of interest}

\section{The authors declare no conflict of interest.}

\section{References}

Schmitt AM, Chang HY. Long noncoding RNAs: at the intersection of cancer and chromatin biology. Cold Spring Harb Perspect Med 2017; 7: a026492.

2. Kour S, Rath PC. Age-related expression of a repeat-rich intergenic long noncoding RNA in the rat brain. Mol Neurobiol 2017; 54: 639-60.

3. Moschovis D, Gazouli M, Tzouvala M, Vezakis A, Karamanolis G. Long non-coding RNA in pancreatic adenocarcinoma and pancreatic neuroendocrine tumors. Ann Gastroenterol 2017; 30: 622-8.

4. Jia W, Chen W, Kang J. The functions of microRNAs and long non-coding RNAs in embryonic and induced pluripotent stem cells. Genomics Proteomics Bioinformatics 2013; 11: 275-83.

5. Yarmishyn AA, Kurochkin IV. Long noncoding RNAs: a potential novel class of cancer biomarkers. Front Genet 2015; 6: 145 .

6. Ricciuti B, Mencaroni C, Paglialunga L, et al. Long noncoding RNAs: new insights into non-small cell lung cancer biology, diagnosis and therapy. Med Oncol 2016; 33: 18.

7. Engreitz JM, Ollikainen N, Guttman M. Long non-coding RNAs: spatial amplifiers that control nuclear structure and gene expression. Nat Rev Mol Cell Biol 2016; 17: 756-70.

8. Ghosal S, Das S, Chakrabarti J. Long noncoding RNAs: new players in the molecular mechanism for maintenance and differentiation of pluripotent stem cells. Stem Cells Dev 2013; 22: 2240-53.

9. Taylor DH, Chu ET, Spektor R, Soloway PD. Long non-coding RNA regulation of reproduction and development. Mol Reprod Dev 2015; 82: 932-56.

10. Jeggari A, Marks DS, Larsson E. miRcode: a map of putative microRNA target sites in the long non-coding transcriptome. Bioinformatics 2012; 28: 2062-3. 
11. Zhu D, Yu Y, Wang W, et al. Long noncoding RNA PART1 promotes progression of non-small cell lung cancer cells via JAK-STAT signaling pathway. Cancer Med 2019; 8: 6064-81.

12. Wu W, Zhao Y, Gao E, et al. LncRNA DLEU2 accelerates the tumorigenesis and invasion of non-small cell lung cancer by sponging miR-30a-5p. J Cell Mol Med 2020; 24: 441-50.

13. Huang N, Guo W, Ren K, et al. LncRNA AFAP1-AS1 supresses miR-139-5p and promotes cell proliferation and chemotherapy resistance of non-small cell lung cancer by competitively upregulating RRM2. Front Oncol 2019; 9: 1103.

14. $\mathrm{Hu} \mathrm{X}$, and Hay JW. First-line pembrolizumab in PD-L1 positive non-small-cell lung cancer: A cost-effectiveness analysis from the UK health care perspective. Lung Cancer 2018; 123: 66-171.

15. Guirgis HM. The impact of PD-L1 on survival and value of the immune check point inhibitors in non-small-cell lung cancer; proposal, policies and perspective. J Immunother Cancer 2018; 6: 15.

16. He Y, Yu H, Rozeboom L, et al. LAG-3 Protein Expression in Non-Small Cell Lung Cancer and Its Relationship with PD-1/PD-L1 and Tumor-Infiltrating Lymphocytes. J Thorac Oncol 2017; 12: 814-23.

17. Wang D, Hu Y. Long Non-coding RNA PVT1 competitively binds MicroRNA-424-5p to regulate CARM1 in radiosensitivity of non-small-cell lung cancer. Mol Ther Nucleic Acids 2019; 16: 130-40.

18. Zhang L, Lin J, Ye Y, et al. Serum MicroRNA-150 Predicts Prognosis for Early-Stage Non-Small Cell Lung Cancer and Promotes Tumor Cell Proliferation by Targeting Tumor Suppressor Gene SRCIN1. Clin Pharmacol Ther 2018; 103: 1061-73.

19. Xu G, Shao G, Pan Q, et al. MicroRNA-9 regulates nonsmall cell lung cancer cell invasion and migration by targeting eukaryotic translation initiation factor 5A2. Am J Transl Res 2017; 9: 478-88.

20. Liu X, Wang M, Cui Y. LncRNA TP73-AS1 interacted with miR-141-3p to promote the proliferation of non-small cell lung cancer. Arch Med Sci 2019; 15: 1547-54.

21. Xu R, Han Y. Long non-coding RNA FOXF1 adjacent non-coding developmental regulatory RNA inhibits growth and chemotherapy resistance in non-small cell lung cancer. Arch Med Sci 2019; 15: 1539-46.

22. Li H, Yin C, Zhang B, et al. PTTG1 promotes migration and invasion of human non-small cell lung cancer cells and is modulated by miR-186. Carcinogenesis 2013; 34: 2145-55

23. Xu Z, Liu D, Fan C, Luan L, Zhang X, Wang E. DIXDC1 increases the invasion and migration ability of non-smallcell lung cancer cells via the PI3K-AKT/AP-1 pathway. Mol Carcinog 2014; 53: 917-25.

24. Sun Y, Zhou Y, Bai Y, et al. A long non-coding RNA HOTTIP expression is associated with disease progression and predicts outcome in small cell lung cancer patients. Mol Cancer 2017; 16: 162.

25. Yu S, Yang D, Ye Y, et al. Long noncoding RNA actin filament-associated protein 1 antisense RNA 1 promotes malignant phenotype through binding with lysine-specific demethylase 1 and repressing HMG box-containing protein 1 in non-small-cell lung cancer. Cancer Sci 2019; 110: 2211-25.

26. Xu M, Xu X, Pan B, et al. LncRNA SATB2-AS1 inhibits tumor metastasis and affects the tumor immune cell microenvironment in colorectal cancer by regulating SATB2. Mol Cancer 2019; 18: 135.
27. Liu SH, Zhu JW, Xu HH, et al. A novel antisense long non-coding RNA SATB2-AS1 overexpresses in osteosarcoma and increases cell proliferation and growth. Mol Cell Biochem 2017; 430: 47-56.

28. Wang YQ, Jiang DM, Hu SS, et al. SATB2-AS1 suppress es colorectal carcinoma aggressiveness by inhibiting SATB2-dependent snail transcription and epithelial-mesenchymal transition. Cancer Res 2019; 79: 3542-56.

29. Guo H, He Y, Bu C, Peng Z. Antitumor and apoptotic ef fects of 5-methoxypsoralen in U87MG human glioma cells and its effect on cell cycle, autophagy and PI3K/ Akt signaling pathway. Arch Med Sci 2019; 15: 1530-8.

30. Ying C, Wang S, Lu Y, et al. Glucose fluctuation increased mesangial cell apoptosis related to AKT signal pathway. Arch Med Sci 2019; 15: 730-7.

31. Yang L, Jiang L, Jiang D, Liu B, Jin S. The protective effects of salvianolic acid A against hepatic ischemia-reperfusion injury via inhibiting expression of toll-like receptor 4 in rats. Arch Med Sci 2019; 15: 1599-607.

32. Eken MK, Ersoy GS, Kaygusuz El, et al. Etanercept protects ovarian reserve against ischemia/reperfusion injury in a rat model. Arch Med Sci 2019; 15: 1104-12.

33. Konecny GE, Winterhoff B, Kolarova T, et al. Expression of $\mathrm{p} 16$ and retinoblastoma determines response to CDK4/6 inhibition in ovarian cancer. Clin Cancer Res 2011; 17: 1591-602.

34. Foster DA, Yellen P, Xu L, Saqcena M. Regulation of G1 cell cycle progression: distinguishing the restriction point from a nutrient-sensing cell growth checkpoint(s). Genes Cancer 2010; 1: 1124-31.

35. Fang H, Zhang S, Guo W, et al. Cobalt protoporphyrin protects the liver against apoptosis in rats of brain death. Clin Res Hepatol Gastroenterol 2015; 39: 475-81.

36. Jiang AJ, Jiang G, Li LT, Zheng JN. Curcumin induces apoptosis through mitochondrial pathway and caspases activation in human melanoma cells. Mol Biol Rep 2015; 42: 267-75.

37. Cui Y, Yi L, Zhao JZ, Jiang YG. Long noncoding RNA HOXA11-AS functions as miRNA sponge to promote the glioma tumorigenesis through targeting miR-140-5p. DNA Cell Biol 2017; 36: 822-8.

38. Xu F, Zhang J. Long non-coding RNA HOTAIR functions as miRNA sponge to promote the epithelial to mesenchymal transition in esophageal cancer. Biomed Pharmacother 2017; 90: 888-96.

39. Yu Y, Zhao JD, Yang H. MiR-299-3p inhibits proliferation and invasion of cervical cancer cell via targeting TCF4. Eur Rev Med Pharmacol Sci 2019; 23: 5621-7.

40. Li Y, Zheng D, Pan L, et al. Knockdown of TUG1 by shRNA inhibited renal cell carcinoma formation by miR-299$3 p / V E G F$ axis in vitro and in vivo. Eur J Pharmacol 2019; 860: 172536.

41. Chen X, Qi M, Yang Q, Li JY. MiR-299-3p functions as a tumor suppressor in thyroid cancer by regulating SHOC2. Eur Rev Med Pharmacol Sci 2019; 23: 232-40.

42. Dang S, Zhou J, Wang Z, Wang K, Dai S, He S. MiR-299-3p functions as a tumor suppressor via targeting Sirtuin 5 in hepatocellular carcinoma. Biomed Pharmacother 2018; 106: 966-75.

43. Li X, Zhang X, Yang C, Cui S, Shen Q, Xu S. The IncRNA RHPN1-AS1 downregulation promotes gefitinib resistance by targeting miR-299-3p/TNFSF12 pathway in NSCLC. Cell Cycle 2018; 17: 1772-83. 